Rev. Int. Contam. Ambie. 36 (3) 729-744, 2020

https://doi.org/10.20937/RICA.53461

\title{
DETERMINACIÓN DE POSIBLE DRENAJE ÁCIDO DE MINA Y CARACTERIZACIÓN DE JALES MINEROS PROVENIENTES DE LA MINA CERRO DE MERCADO, DURANGO, MÉXICO
}

Determination of possible acid mine drainage and characterization of mine tailings from the

Cerro de Mercado mine", Durango, Mexico

\author{
Fernando Benigno SALAS URVIOLA ${ }^{1,2 *}$, Pedro GUADARRAMA GUZMÁN ${ }^{2}$, \\ José Juan GUTIÉRREZ BAZÁN², Nadia GARCÍA LUNA², Georgina FERNÁNDEZ VILLAGÓMEZ², \\ y María Teresa ALARCÓN HERRERA ${ }^{3}$
}

${ }^{1}$ Facultad de Ingeniería de Minas, Universidad Nacional del Altiplano Puno, Apartado Postal 291, Puno, Perú

${ }^{2}$ Laboratorio de Ingeniería Sanitaria y Ambiental, Facultad de Ingeniería, Universidad Nacional Autónoma de México, Circuito Escolar s/n, Ciudad Universitaria, 04510 Ciudad de México, México

${ }^{3}$ Centro de Investigación en Materiales Avanzados, sede Durango, Calle CIMAV 110, Ejido Arroyo Seco, Colonia 15 de Mayo (Tapias), 34147 Durango, México

*Autor para correspondencia: bensalas7@hotmail.com

Palabras clave: ambiente, actividades antrópicas, metales pesados, alcalinidad, lixiviados

\section{RESUMEN}

La mina Cerro de Mercado, ubicada en el estado de Durango, México, ha almacenado jales durante el proceso de extracción de hematita y magnetita durante aproximadamente 80 años. La presente investigación tuvo como objetivo llevar caracterizar los jales de la presa Boleo Estrella de la mina Cerro de Mercado y determinar el potencial de generación de drenaje ácido, considerando la Norma Oficial Mexicana NOM141-SEMARNAT-2003. El muestreo se realizó de acuerdo con la Norma Mexicana NMX-AA-132-SCFI-2006 y se obtuvieron 27 muestras en 16.2 ha de la superficie de la presa. Como resultado de la caracterización de dichos residuos, se obtuvieron valores de $\mathrm{pH}$ alcalinos y concentraciones mínimas de azufre y sulfatos; la relación potencial de neutralización/potencial de acidez fue mayor a 1.2, lo que indica que no son generadores potenciales de drenaje ácido. Por otro lado, la determinación de metales y metaloides considerados elementos potencialmente tóxicos presentes en las muestras de jales, evidencian la presencia de arsénico (As) y bario (Ba) en valores inferiores a los límites máximos permisibles (LMP) indicados en la NOM-157 SEMARNAT-2009; asimismo, el $22.2 \%$ de dichas muestras está dentro de los LMP establecidos para cromo (Cr) y el $77.8 \%$ se halla ligeramente por encima de los LMP. En el $66.7 \%$ de los jales muestreados no se detectó antimonio (Sb); sin embargo, el $33.3 \%$ restante contiene $\mathrm{Sb}$ en cantidades superiores a los LMP. De acuerdo con el estudio realizado, se puede deducir que los jales analizados no generan drenaje ácido y en las condiciones ambientales actuales, aun con la presencia de $\mathrm{Cr}$ y $\mathrm{Sb}$, no presentan peligro para el ambiente.

Key words: environment, anthropic activities, heavy metals, alkalinity, leachate

\begin{abstract}
The Cerro de Mercado mine, located in the state of Durango, Mexico, has stored tailings during the extraction process of hematite and magnetite for approximately 80 years. The objective of the present investigation was to characterize the tailings of the
\end{abstract}


Boleo Estrella dam of the Cerro de Mercado mine, considering the Official Mexican Standard NOM-141-SEMARNAT-2003. The sampling was carried out in accordance with the Mexican Standard NMX-AA-132-SCFI-2006. Twenty-eight samples were obtained considering 16.2 ha of the dam surface. As a result of the residues characterization, alkaline $\mathrm{pH}$ values and minimum concentrations of sulfur and sulfates were obtained; the ratio of neutralization potential/acidity potential was greater than 1.2 , which indicates they are not potential generators of acid drainage. On the other hand, the determination of metals and metalloids present in the tailings samples, which are considered potentially toxic elements, show the presence of arsenic (As) and barium (Ba) in values below the maximum permissible limits (MPL) indicated in NOM-157 SEMARNAT-2009; likewise, $22.2 \%$ of these samples are within the established MPL for chromium $(\mathrm{Cr})$ and $77.8 \%$ are slightly above it. On the other hand, antimony $(\mathrm{Sb})$ was not detected in $66.7 \%$ of the tailings sampled; however, the remaining $33.3 \%$ contains levels of Sb higher than the MPL. According to our study, it can be deduced that the tailings analyzed do not generate acid drainage and under the current environmental conditions, even with the presence of $\mathrm{Cr}$ and $\mathrm{Sb}$, they do not pose a danger to the environment.

\section{INTRODUCCIÓN}

Los problemas ambientales causados por la explotación minera han aumentado significativamente por la liberación de diversos elementos químicos en el entorno circundante (aire, agua y suelo) (Navarro et al. 2008, Varrica et al. 2014, Zeng et al. 2018). Asimismo, estas actividades minero-metalúrgicas generan cantidades considerables de residuos denominados jales, que contienen metales como sulfuros (Ye et al. 2017) y fluidos o lodos de procesamiento (Kossoff et al. 2014), los cuales son restos de grano fino que se depositan sin tratamiento en presas de jales (Entezari-Zarandi et al. 2017, Bedi et al. 2018). Los sulfuros se oxidan en contacto con el agua, lo que reduce el $\mathrm{pH}$ circundante y produce lixiviación de los óxidos (Kiventera et al. 2018). Los óxidos pueden migrar como partículas suspendidas o ser retenidos en los residuos, y los metales pesados muestran afinidad con los óxidos de hierro en el ambiente (Zhang et al. 2018). Históricamente, los planes de manejo de jales se han enfocado al confinamiento y contención sin tener en cuenta la alteración de sus propiedades físicas y químicas a largo plazo (Santini y Banning 2016), lo que pone en riesgo al ecosistema y la salud humana local (Schoenberger 2016, Ye et al. 2017). Muchos países han adoptado medidas para reducir la contaminación ambiental, emitiendo legislación sobre la industria minera para el mejoramiento y la conservación del ambiente (Awan 2013, Elvan 2013, Zeng et al. 2018), el manejo sostenible de sus residuos y la responsabilidad social (Tost et al. 2018), con lo cual se evitan conflictos sociales que a menudo son muy complejos (Fraser 2018).
En México, la minería ha sido una de las actividades económicas más importantes desde la época prehispánica hasta la actualidad (Salinas-Rodríguez et al. 2016); sin embargo, a pesar de que ofrece grandes beneficios para la economía del país, también es una fuente de contaminación ambiental (Manrique-Ramírez y Rodríguez-Rosales 2015) que genera una gran cantidad de residuos (jales, relaves o colas) (Romero et al. 2008) y sitios contaminados a lo largo de todo el territorio, los cuales se ubican principalmente en Chihuahua, Michoacán, Zacatecas, Durango, Sonora, Coahuila, Guanajuato, San Luis Potosí, Hidalgo, Sinaloa, Colima y Jalisco (Volke y Velasco 2002, Guzmán, 2016, Covarrubias y Peña 2017). Por ello, uno de los grandes retos de la minería mexicana es la sana convivencia con las comunidades y el ambiente (CAMIMEX 2017).

La mina Cerro de Mercado, uno de los yacimientos de hierro más importantes de México, ha sido explotada desde 1828 para satisfacer las necesidades de la industria siderúrgica del país. El yacimiento está emplazado en una secuencia de rocas volcánicas del terciario compuesto por una brecha cuyos fragmentos son de riodacita cementada con hematita; mineralógicamente, está constituido por magnetita $\left(\mathrm{Fe}^{2+}\right.$, $\left.\mathrm{Fe}^{3+}\right)_{2} \mathrm{O}_{4}$ y hematita $\left(\mathrm{Fe}_{2} \mathrm{O}_{3}\right)$ (Corona-Esquivel et al. 2003). Actualmente se explotan diferentes cuerpos de mena, que por sus características se han denominado mineral masivo, pulverulento, brechas mineralizadas y cuerpos rodados de hierro semiconsolidados. $\mathrm{Su}$ producción aproximada es de 1.1 millones de toneladas por año con leyes del 25 al $47 \%$ de Fe y una recuperación del $38 \%$ (Corona-Esquivel et al. 2009, 2016). 
El drenaje ácido de mina (DAM) -agua contaminada por la oxidación de sulfuros metálicos- tiene típicamente un $\mathrm{pH}$ ácido y niveles elevados de sulfato y metales pesados que se dispersan en el entorno (Rashed 2010, SEMARNAT 2011, Sgier et al. 2015, Kastyuchik et al. 2017, Pavoni et al. 2018), lo que ocasiona problemas de contaminación ambiental a nivel local (Johnson y Hallberg 2005, Vasilatos et al. 2015, Nleya et al. 2016, Bwapwa et al. 2017, Kefeni et al. 2017, Pape et al. 2017). Su generación, separación, movilidad y mitigación abarcan procesos complejos que implican la combinación de factores físicos, químicos y biológicos (Simate y Ndlovu 2014, Gitari et al. 2018).

Aduvire (2006) resalta que el tamaño de las partículas influye en la velocidad de reacción, la cual aumenta a medida que disminuye el tamaño (aumenta la superficie específica), aunque también reduce la infiltración y la entrada de aire; asimismo, indica que los principales elementos que intervienen en la formación de DAM son sulfuros, oxígeno y agua. Las presas de relaves o jales tienen una granulometría uniforme al $100 \%$ con un tamaño inferior a $0.2 \mathrm{~mm}$; también tienen capacidad de compactación y su baja permeabilidad constituye una barrera a la difusión del agua y el oxígeno. Por otro lado, este autor menciona que el agua y el oxígeno son dos componentes esenciales en la reacción, y la exclusión de cualquiera de ellos paralizaría el proceso de formación de aguas ácidas.

La presente investigación tuvo como objetivo caracterizar jales de la presa Boleo Estrella de la mina Cerro de Mercado y determinar el potencial de capacidad de generación de drenaje ácido, considerando la norma NOM-141-SEMARNAT-2003.

\section{MATERIALES Y MÉTODOS}

\section{Área de estudio}

El yacimiento de Cerro de Mercado se localiza en el centro del estado de Durango, justo en el límite norte de la actual capital, Victoria de Durango (Fig. 1).

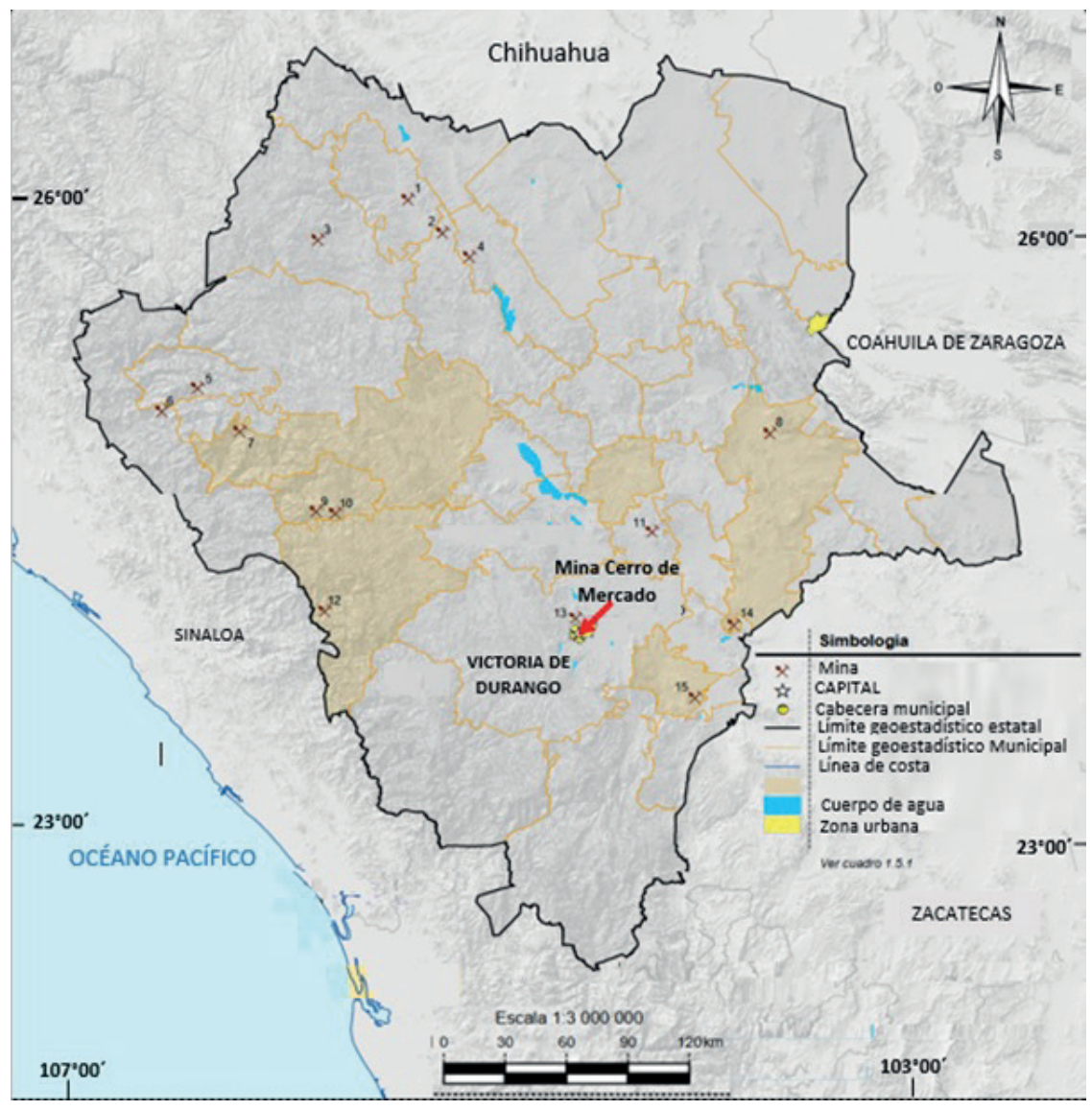

Fig. 1. Ubicación de la mina Cerro de Mercado ( $24^{\circ} 03^{\prime} 24^{\prime}$ ' N y $104^{\circ} 40^{\prime} 12^{\prime}$ W) (INEGI, 2017) 
Sus coordenadas geográficas son $24^{\circ} 03^{\prime} 24^{\prime \prime} \mathrm{N}$ y $104^{\circ}$ 40 ' 12" W, y su altitud es de $1980 \mathrm{msnm}$. La mineralogía de la mena consiste principalmente de hematita $\left(\mathrm{Fe}_{2} \mathrm{O}_{3}\right)$ y magnetita $\left(\mathrm{Fe}_{3} \mathrm{O}_{4}\right)$. En menor proporción, junto con la magnetita aparecen, como minerales primarios, cristales euhedrales de piroxeno y apatita intercrecidos con la magnetita (Corona-Esquivel et al. 2009). En Durango el $40 \%$ del territorio presenta clima seco y semiseco, el $34 \%$ tiene clima templado subhúmedo, el $14 \%$ presenta clima muy seco, el $11 \%$ es cálido subhúmedo y el restante $1 \%$ es templado húmedo (INEGI 2010).

\section{Muestreo}

El plan de muestreo se realizó de acuerdo con la norma mexicana NMX-AA-132-SCFI-2006 (SE 2006). Se tomaron 27 muestras considerando el área de 16.2 ha que abarca la presa Boleo Estrella. Se intentó obtener dichas muestras de manera equidistante, pero debido a la densa vegetación en algunas partes no fue posible (Fig. 2). Se tomaron a una profundidad de $30 \mathrm{~cm}$; los puntos de recolección se referenciaron mediante un posicionador geográfico satelital (GPS). Los jales se recolectaron con las herramientas que marca la norma mexicana mencionada y se guardaron en bolsas de polietileno selladas y etiquetadas para su posterior identificación, teniendo cuidado de cumplir la cadena de custodia hasta su llegada al Laboratorio de Ingeniería Sanitaria y Ambiental de la Facultad de Ingeniería de la Universidad Nacional Autónoma de México (LISA-FI-UNAM).

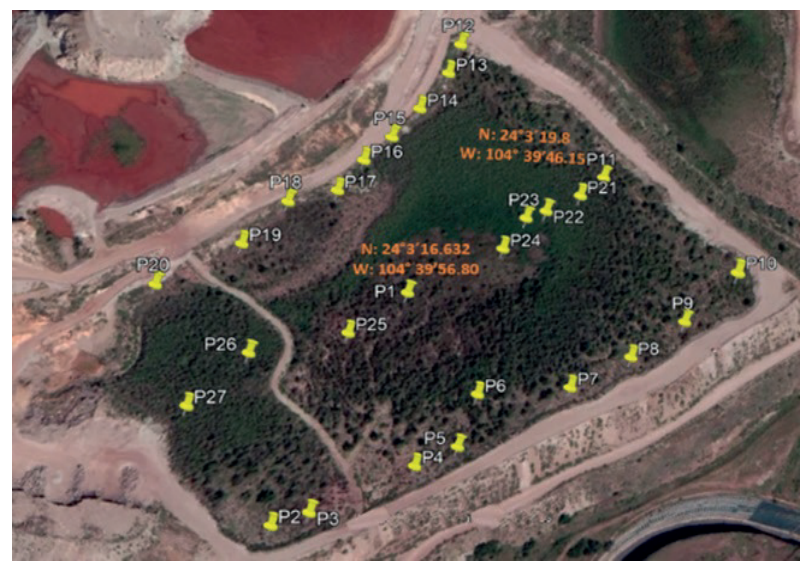

Fig. 2. Puntos de muestreo en la presa Boleo Estrella, mina Cerro de Mercado, Durango

\section{Caracterización}

La preparación de las muestras de jales consistió en secarlas a temperatura ambiente en el laboratorio para su posterior caracterización. Para comprobar si son potenciales generadores de DAM se efectuaron la medición del pH (SE 2012) y la prueba modificada de balance ácido-base (ABA) (Sobek et al. 1978, Lawrence y Wang 1996, SEMARNAT 2004). Con el fin de saber si los jales de la mina de estudio generan drenaje ácido se determinaron los siguientes parámetros: $a$ ) potencial de neutralización $(\mathrm{PN})$, el cual consta de la medición cualitativa de carbonatos. Dicho procedimiento se realizó mediante la adición de $\mathrm{HCl}$ al $25 \%$ a las muestras, observándose una efervescencia fuerte debida a la reacción entre los carbonatos presentes en los jales y el ácido agregado, y b) medición del poder de neutralización, que consiste en agregar $\mathrm{HCl} 1 \mathrm{~N}$ a las muestras en diferentes tiempos de acuerdo con lo señalado en la tabla 1 , anexo 5, de la NOM-141-SEMARNAT 2003, y posteriormente titularlas con $\mathrm{NaOH}$ 0.1 N hasta alcanzar un $\mathrm{pH}$ de 8.3.

El potencial de neutralización (PN) se calculó expresado en $\mathrm{kg}_{\mathrm{CaCO} 3} / \mathrm{t}$ de jales utilizando la siguiente formula:

$$
P N=\frac{(0.1 \times \text { vol en } m L \text { de } \mathrm{NaOH})}{\text { peso de la muestra }}
$$

donde $V_{\mathrm{f}}$ representa los mililitros de $\mathrm{HCl}$ utilizados.

El potencial de acidez (PA) consiste en la determinación de azufre en forma de sulfatos y azufre total. Para la determinación del azufre en forma se sulfatos se utilizó el método turbidimétrico (APHA 2003), el cual determina los sulfatos en $\mathrm{mg} / \mathrm{L}$ de concentraciones menores a $1 \mathrm{mg}$ de $\mathrm{SO}_{4}{ }^{2-} / \mathrm{L}$. El ion sulfato se precipita en un medio ácido $(\mathrm{HCl})$ con cloruro de bario $\left(\mathrm{BaCl}_{2}\right)$ y forma cristales de $\mathrm{Ba}$ de tamaño uniforme; a continuación se mide la absorbancia y se determina la concentración del ion sulfato comparándola con la curva patrón de calibración previamente realizada. Para la determinación se utilizó un equipo turbidimétrico marca Hach $2100 \mathrm{~N}$ :

$m g \mathrm{SO}_{4}^{2-} / L=\frac{m g \mathrm{SO}_{4}^{2-} \times 1000}{m l \text { de muestra }}$

Por otro lado, el análisis del azufre total se realizó en el Laboratorio de Química Analítica del Instituto de Geofísica de la Universidad Nacional Autónoma de México, utilizando un equipo Leco S-144 DR Dual Range Sulfur Analyzer, el cual se calibró con el estándar Sulfur $1.09 \% \pm 0.05 \%$ Sulfur in Ccall por el método infrarrojo. Con la determinación de azufre en forma de sulfatos y azufre total se determinó el potencial de acidez. Para los cálculos se utilizó la siguiente fórmula (SEMARNAT 2004): 
$\% S^{2-}=\left(\% S_{\text {total }}-\% S_{\text {sulfatos }}\right)$

$P A=\% S^{2-} \times 31.25$

Asimismo, se cuantificóla concentración de metales y metaloides considerados elementos potencialmente tóxicos por el método de fluorescencia de rayos $\mathrm{X}$ utilizando el equipo Pistola Nitton XL3t Ultra. La empresa Thermo Scientific realizó la curva de calibración, y el laboratorio de Geoquímica Ambiental del Instituto de Geología llevó a cabo la verificación al principio y al final de cada día del análisis para 20 muestras, usando material estándar de referencia Montana 2710a con un criterio de aceptación del equipo portátil de $\pm 20 \%$ de exactitud (USEPA 2007).

\section{Estado de oxidación del cromo}

La determinación del cromo presente en las muestras de la presa de jales Boleo Estrella se llevó a cabo mediante la técnica de digestión alcalina para cromo hexavalente registrada en la NOM-147-SEMARNAT/SSA1-2004 (SEMARNAT 2007). En dicha técnica el $\mathrm{Cr}^{6+}$ se reduce a $\mathrm{Cr}^{3+}$ mediante la adición de difenilcarbazida, y ésta se oxida a difenilcarbazona, reacción redox caracterizada por el desarrollo de un color violeta. En la figura 3 se observan algunas etapas de este procedimiento.

\section{Pruebas de movilidad}

Las pruebas de movilidad de cromo y antimonio se llevaron a cabo mediante el método de lixiviación con agua en equilibrio en $\mathrm{CO}_{2}$ establecido en la NOM-141-SEMARNAT-2003. Las concentraciones de cromo en el extracto se determinaron mediante absorción atómica en un equipo GBC Avanta y las concentraciones de antimonio mediante la técnica de espectroscopia de masas por plasma acoplado inductivamente (ICPS-MS). En la figura 4 se observan tanto la prueba de extracción como los equipos utilizados.
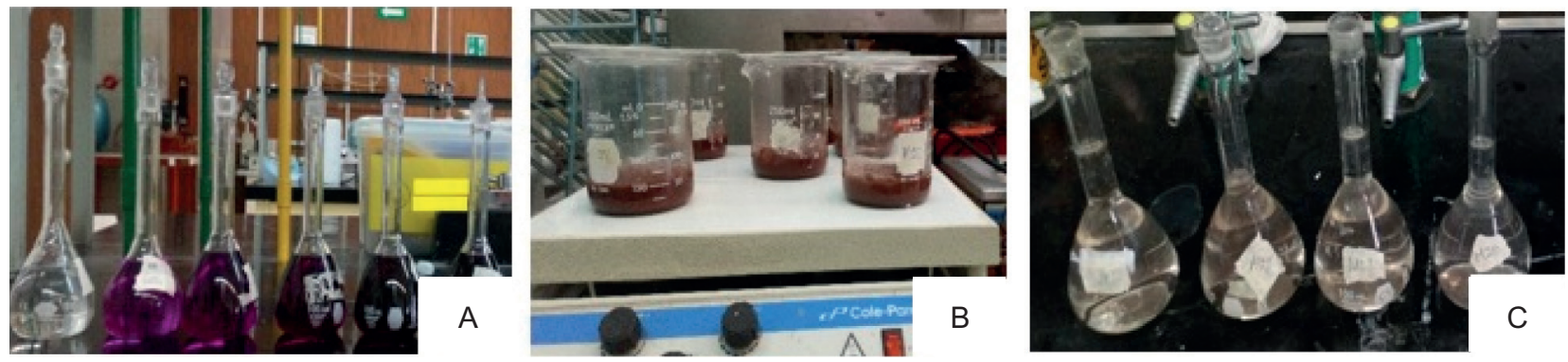

Fig. 3. (a) Curva de calibración, (b) muestras en el transcurso de la prueba y (c) muestras listas para ser leídas en el espectrofotómetro
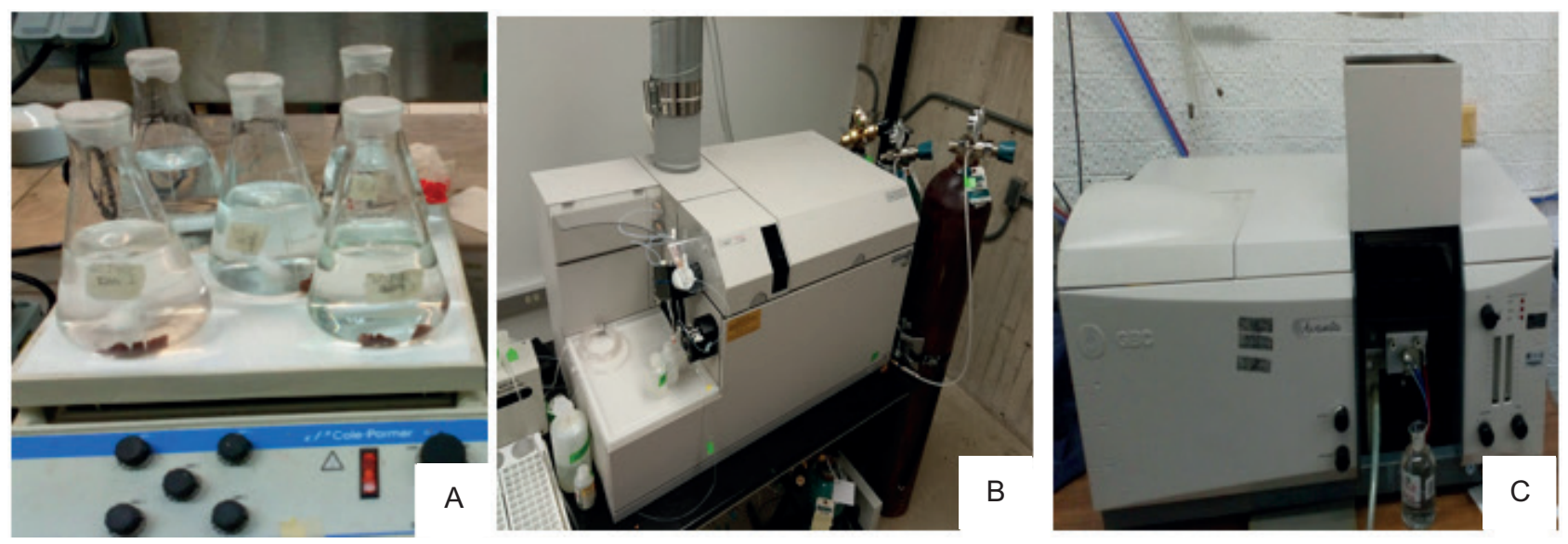

Fig. 4. (a) Extracción, (b) equipo de espectrometría de masas con plasma acoplado inductivamente utilizado y (c) equipo de absorción atómica 
CUADRO I. RESULTADOS DE LA GRANULOMETRIA DE JALES

\begin{tabular}{rcccccc}
\hline \# Malla & $\begin{array}{c}\text { Apertura } \\
(\mathrm{mm})\end{array}$ & $\begin{array}{c}\text { Masa retenida } \\
(\mathrm{g})\end{array}$ & $\begin{array}{c}\text { \% retenido } \\
(\mathrm{g})\end{array}$ & $\begin{array}{c}\text { \% retenido } \\
\text { (acumulado) }\end{array}$ & $\begin{array}{c}\% \\
\text { pasa }\end{array}$ & $\begin{array}{c}\text { Módulo de } \\
\text { finura }\end{array}$ \\
\hline 8 & 2.36 & 14.61 & 2.92 & 2.92 & 97.33 & 2.7 \\
16 & 1.18 & 26.01 & 5.20 & 8.12 & 92.177 & \\
30 & 0.6 & 37.67 & 7.53 & 15.66 & 84.33987076 & \\
50 & 0.3 & 69.91 & 13.98 & 29.64 & 70.35598368 & \\
100 & 0.15 & 128.14 & 25.63 & 55.28 & 44.72397483 & \\
200 & 0.075 & 16.93 & 3.4 & 58.66 & 41.33646502 & \\
$>200$ & -- & 206.65 & 41.34 & 100.00 & & \\
\hline
\end{tabular}

Potencial de hidrógeno $(\mathrm{pH})$

\section{RESULTADOS Y DISCUSIÓN}

\section{Granulometría de los jales}

La granulometría de los jales de la presa Boleo Estrella se determinó de acuerdo a la norma ASTM C 136-01 Standard Test Method for Sieve Analysis of Fine and Coarse Aggregates, donde establece que los valores de módulo de finura de 2.5 a 3 son normales para un agregado fino. En el cuadro I, el resultado del valor de módulo de finura es 2.7 de los jales de la presa Boleo Estrella, obtenido aplicando la fórmula de sumatoria de $\%$ retenido (acumulado) dividido entre 100, por lo tanto, se clasifican como agregados ligeramente finos, es preciso señalar que Mena y Loera (1972) aplicaron la norma ASTM C136-01 para la obtención del valor de módulo de finura.

En la figura 5 se presentan los resultados de la determinación del $\mathrm{pH}$. Se aprecian valores alcalinos en todas las muestras de jales, exponiendo en primera instancia que no son generadores potenciales de drenaje ácido de mina. El $\mathrm{pH}$ promedio de las muestras es de 9.07.

\section{Potencial de neutralización (PN)}

De los valores obtenidos por la medición cualitativa de carbonatos en las 27 muestras (Cuadro II), 25 calificaron como fuertes (93\%) y dos como moderados (7\%). Lo anterior se efectuó de acuerdo con lo establecido en la NOM-141-SEMARNAT-2003, anexo 5, punto 4.4.1, en el cual se establecen cuatro grados de reacción: nulo, bajo, moderado y fuerte. El hecho de que las muestras de jales califiquen como fuertes indica un gran contenido de carbonatos presentes. Con esta primera prueba se tiene un indicio de que poseen capacidad suficiente para neutralizar a los sulfuros.

El cálculo del poder de neutralización se efectuó con base en la NOM-141-SEMARNAT 2003. En la figura 6 se puede observar que estas muestras de jales tienen un poder neutralizador de 64 a

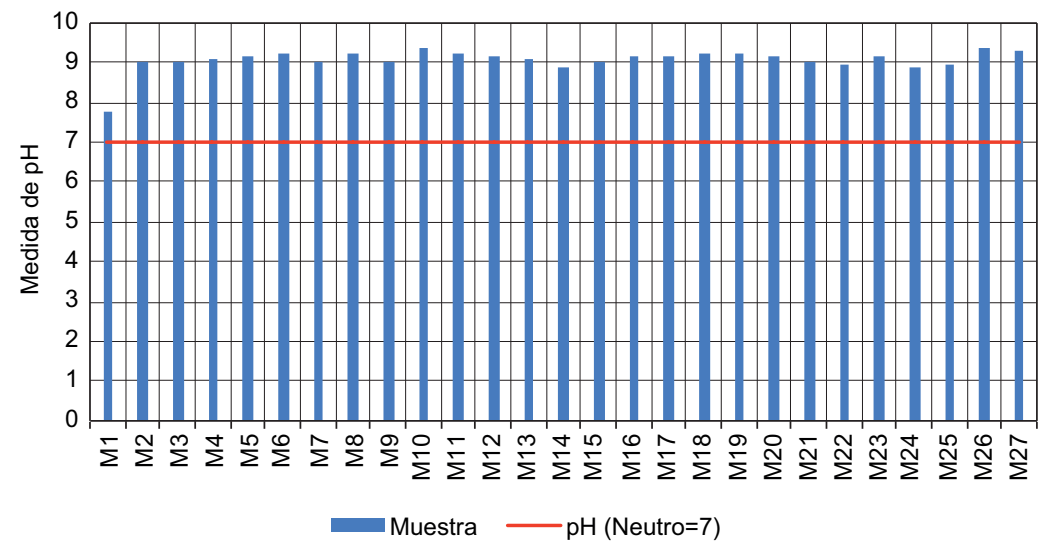

Fig. 5. Resultado de la determinación de potencial de hidrógeno $(\mathrm{pH})$ en las muestras de jales 
CUADRO II. RESULTADOS DE LA REACCIÓN DE CARBONATOS EN MUESTRAS DE JALES

\begin{tabular}{cccccc}
\hline Muestra & Calificativo & Muestra & Calificativo & Muestra & Calificativo \\
\hline M-1 & Fuerte & M-10 & Fuerte & M-19 & Fuerte \\
M-2 & Fuerte & M-11 & Fuerte & M-20 & Fuerte \\
M-3 & Fuerte & M-12 & Fuerte & M-21 & Fuerte \\
M-4 & Fuerte & M-13 & Fuerte & M-22 & Fuerte \\
M-5 & Fuerte & M-14 & Fuerte & M-23 & Fuerte \\
M-6 & Fuerte & M-15 & Fuerte & M-24 & Fuerte \\
M-7 & Fuerte & M-16 & Fuerte & M-25 & Fuerte \\
M-8 & Fuerte & M-17 & Fuerte & M-26 & Moderado \\
M-9 & Fuerte & M-18 & Fuerte & M-27 & Moderado \\
\hline
\end{tabular}

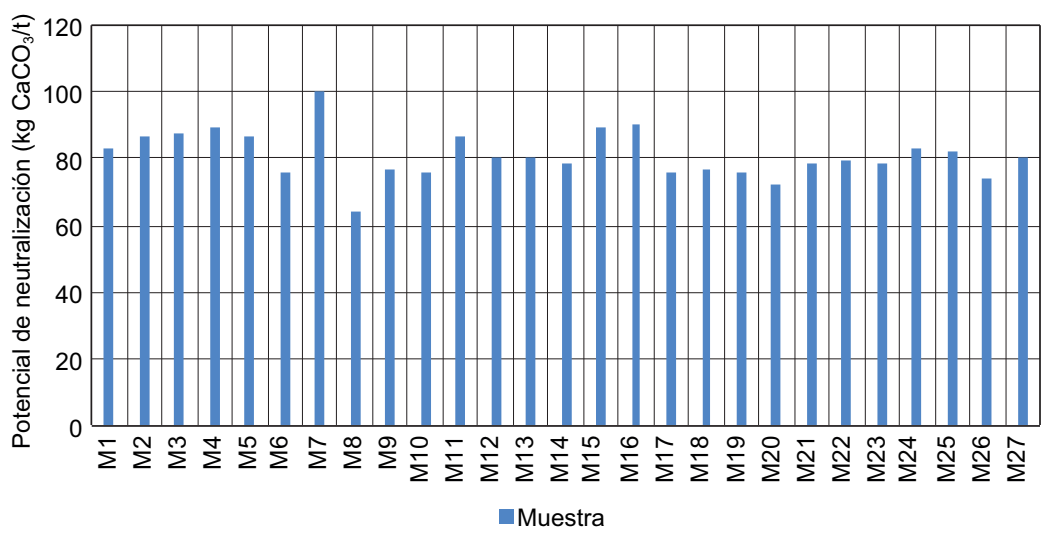

Fig. 6. Resultados de determinación del potencial de neutralización en las muestras de jales

$100 \mathrm{~kg}$ de $\mathrm{CaCO}_{3} / \mathrm{t}$. La muestra M-8 presentó el PN más bajo con $64 \mathrm{~kg} \mathrm{CaCO}_{3} / \mathrm{t}$, mientras que la M-7 tuvo un PN de $100 \mathrm{~kg} \mathrm{CaCO}_{3} / \mathrm{t}$. Para calcular el PN expresado como $\mathrm{kg} \mathrm{CaCO}_{3} / \mathrm{t}$ en los jales se aplicó la fórmula 1.

\section{Potencial de acidez}

Por el método turbidimétrico se determinó el azufre en forma de sulfatos. Los resultados se aprecian en el cuadro III. Los cálculos se ejecutaron con la fórmula 2. La determinación del porcentaje de azufre total se realizó en el Laboratorio de Química Analítica del Instituto de Geofísica de la UNAM con un equipo Leco S-144 DR Dual Range Sulfur Analyzer, el cual se calibró con el estándar Sulfur 1.09\% $\pm 0.05 \%$ Sulfur in Ccall, por el método infrarrojo. Los resultados indican no detectable con límite de detección del equipo al $1 \%$ de azufre total. Para efectos de cálculo del $\% S_{\text {total, }}$, cuando los equipos evidencian un resultado no detectable se utiliza por convención y con propósitos estadísticos la mitad del límite de detección del equipo (en este caso $0.5 \%$ ).
Así, asumiendo por convención $0.5 \%$ de $S_{\text {total }}$ y con los resultados obtenidos de azufre en forma de sulfatos en cada una de las muestras, se calculó el PA de acuerdo con la norma NOM-141-SEMARNAT 2003 (Fig. 7), usando las fórmulas 3 y 4.

La NOM-141-SEMARNAT 2003 establece los siguientes valores de la relación PN/PA; si ésta es $\leq 1.2$, los jales son potenciales generadores de drenaje ácido; si es $>1.2$ los jales no son potenciales generadores de drenaje ácido. En la figura 8 se observa que la relación PN/PA para todas las muestras es superior a 1.2, por lo que de acuerdo con la norma antes mencionada los jales de la presa Boleo Estrella no son potenciales generadores de drenaje ácido. Por otro lado, la NOM-157-SEMARNAT-2009 establece los límites para determinar la peligrosidad debida al potencial de generación de drenaje ácido: si la relación PN/PA > 3 no generará drenaje ácido, por lo que se califica como no peligroso. Basándonos en ambas normas se puede concluir que los jales de la presa antes mencionada no constituyen un peligro. 
CUADRO III. RESULTADOS DE DETERMINACIÓN DEL PORCENTAJE DE SULFATOS EN MUESTRAS DE JALES

\begin{tabular}{clclcl}
\hline Muestra & \% sulfatos & Muestra & \% sulfatos & Muestra & \% sulfatos \\
\hline M-1 & 0.00125 & M-10 & 0.004916667 & M-19 & 0.005083333 \\
M-2 & 0.0004 & M-11 & 0.001 & M-20 & 0.020916667 \\
M-3 & 0.010333333 & M-12 & 0.0205 & M-21 & 0.007 \\
M-4 & 0.001666667 & M-13 & 0.025333333 & M-22 & 0.008666667 \\
M-5 & 0.004166667 & M-14 & 0.000166667 & M-23 & 0.013 \\
M-6 & 0.006333333 & M-15 & 0.000333333 & M-24 & 0.024 \\
M-7 & 0.00775 & M-16 & 0.008583333 & M-25 & 0.007416667 \\
M-8 & 0.002916667 & M-17 & 0.024916667 & M-26 & 0.015333333 \\
M-9 & 0.000333333 & M-18zY & 0.020833333 & M-27 & 0.0005 \\
\hline
\end{tabular}

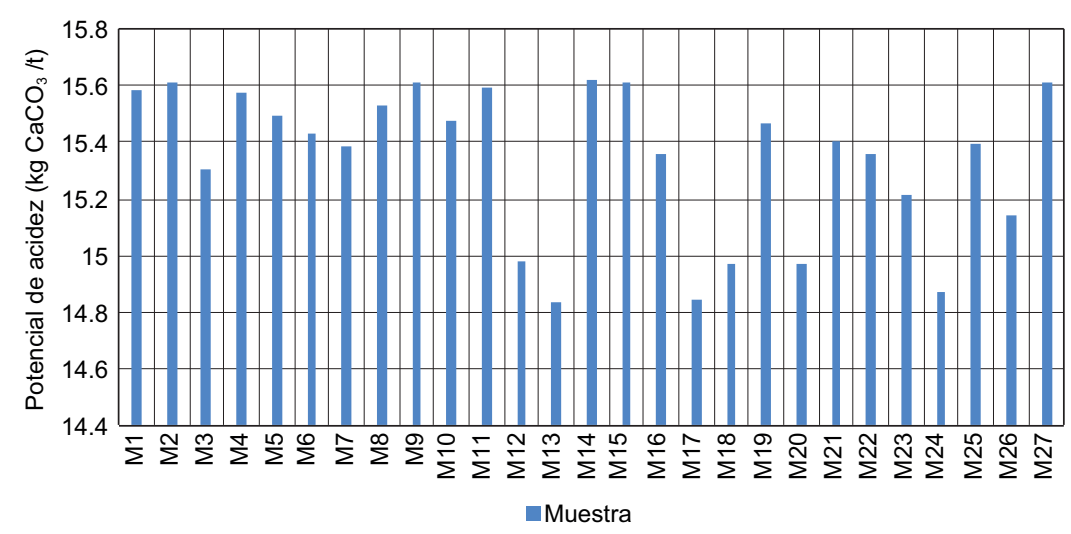

Fig. 7. Resultados del potencial de acidez de las muestras de jales

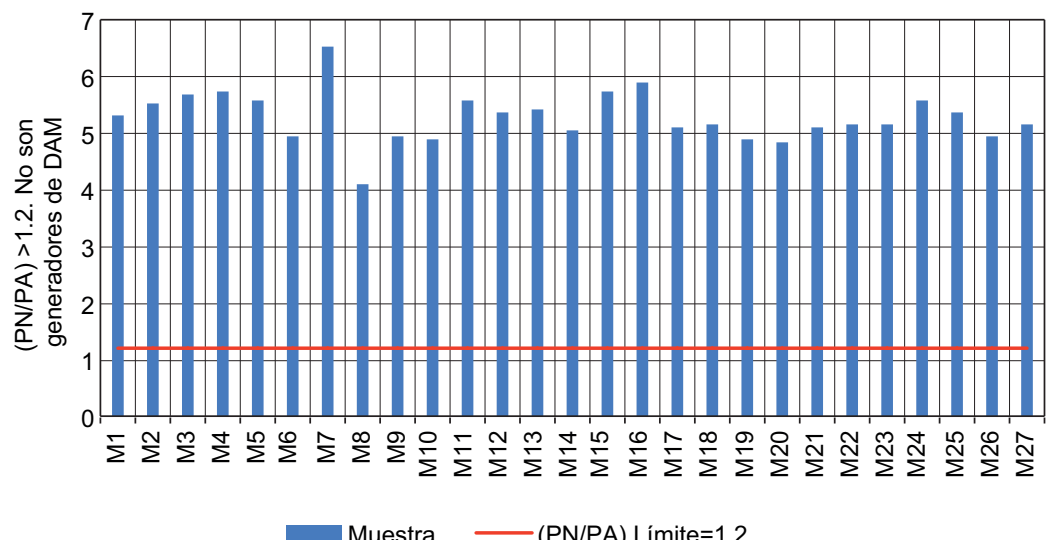

Fig. 8. Relación de potencial de neutralización/potencial de acidez (PN/PA) de muestras de jales que no son generadores de drenaje ácido de mina (DAM)

\section{Metales y metaloides}

La determinación de los metales y metaloides presentes en las muestras de jales se realizó en el Laboratorio Nacional de Geoquímica y Mineralogía del Instituto de Geología de la UNAM, con el equipo Pistola Nitton XL3t Ultra, mediante análisis semi- cuantitativo por fluorescencia de rayos $\mathrm{X}$ (método US-EPA 6200). El análisis de las muestras indica que hay presencia de los siguientes metales: $\mathrm{Zr}, \mathrm{Sr}, \mathrm{Rb}, \mathrm{As}$, $\mathrm{Zn}, \mathrm{Cu}, \mathrm{Mn}, \mathrm{Ti}, \mathrm{Fe}, \mathrm{Ni}, \mathrm{Cr}, \mathrm{V}, \mathrm{Ba}, \mathrm{Sb}$ y Ca. Los metales que se encuentran por debajo del límite de detección son $\mathrm{Pb}, \mathrm{W}, \mathrm{Mo}, \mathrm{U}, \mathrm{Tl}, \mathrm{Au}, \mathrm{Se}, \mathrm{Hg}, \mathrm{Co}, \mathrm{Cd}$ y Pd. 
Algunos metales como el cromo y antimonio están por encima del límite máximo permisible establecido por la norma NOM-157-SEMARNAT-2009 (10.6 $\mathrm{mg} / \mathrm{kg}$ para antimonio y $100 \mathrm{mg} / \mathrm{kg}$ para cromo).

Los resultados de laboratorio señalan un valor de
As inferior al LMP de $100 \mathrm{mg} / \mathrm{kg}$ en las 27 muestras de jales (Fig. 9); el Ba está por debajo del LMP(Fig. 10) y el $\mathrm{Cr}$ se encuentra en todas las muestras como $\mathrm{Cr}$ total (Fig. 11). Por último, con la pistola Nitton no se detectó $\mathrm{Sb}$ en 18 de 27 muestras $(66.7 \%$ ), y en las

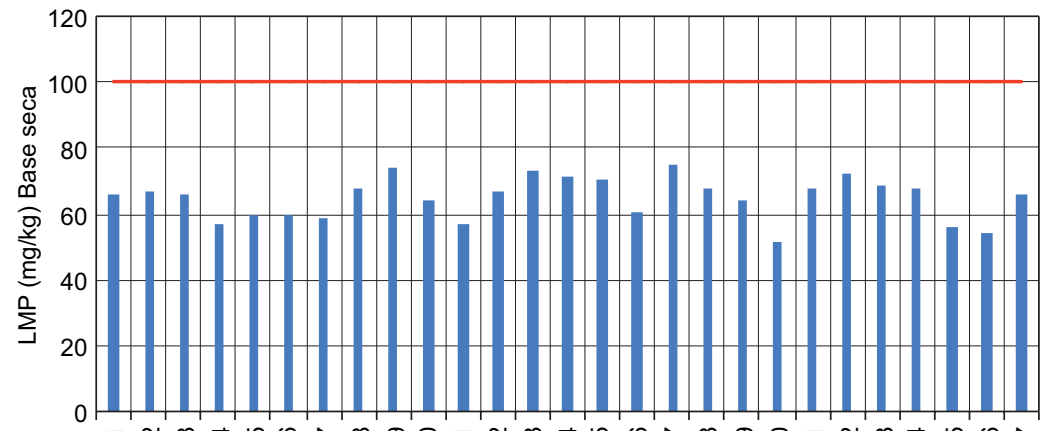

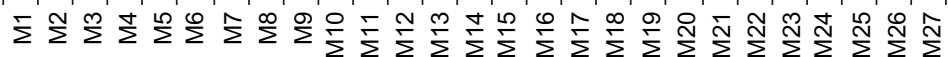

Muestra L LMP $=100 \mathrm{mg} / \mathrm{kg}$

Fig. 9. Comparación de los resultados de la determinación de arsénico en muestras de jales con el límite máximo permisible (LMP)

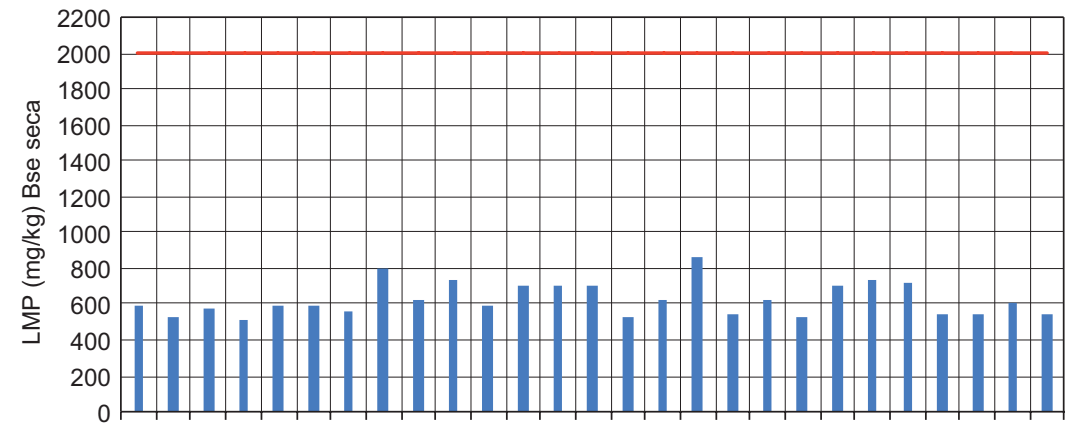

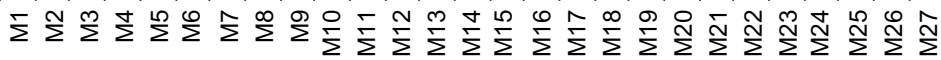

Muestra L LMP $=2000 \mathrm{mg} / \mathrm{kg}$

Fig. 10. Comparación de los resultados de la determinación de bario en muestras de jales con el límite máximo permisible (LMP)

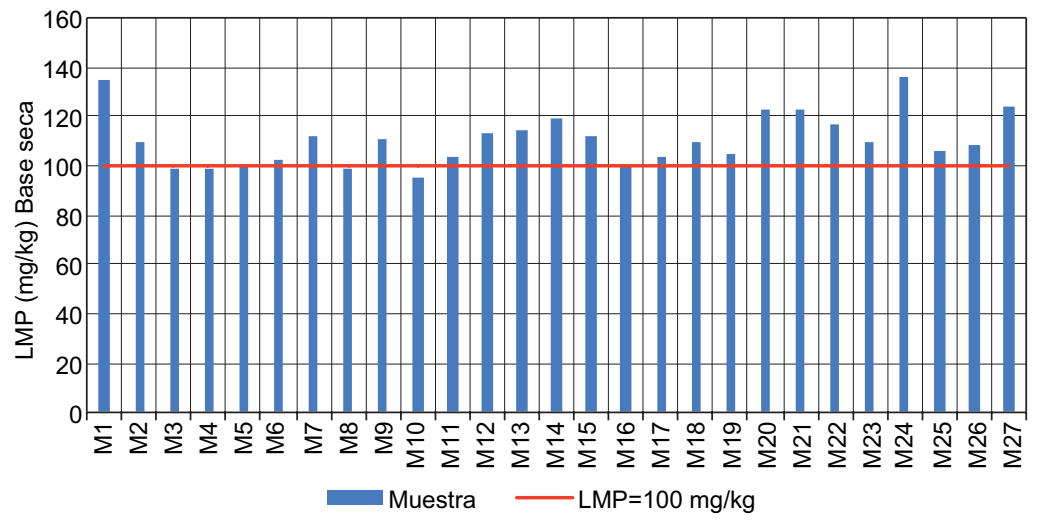

Fig. 11. Comparación de los resultados de la determinación de cromo en muestras de jales con el límite máximo permisible (LMP) 


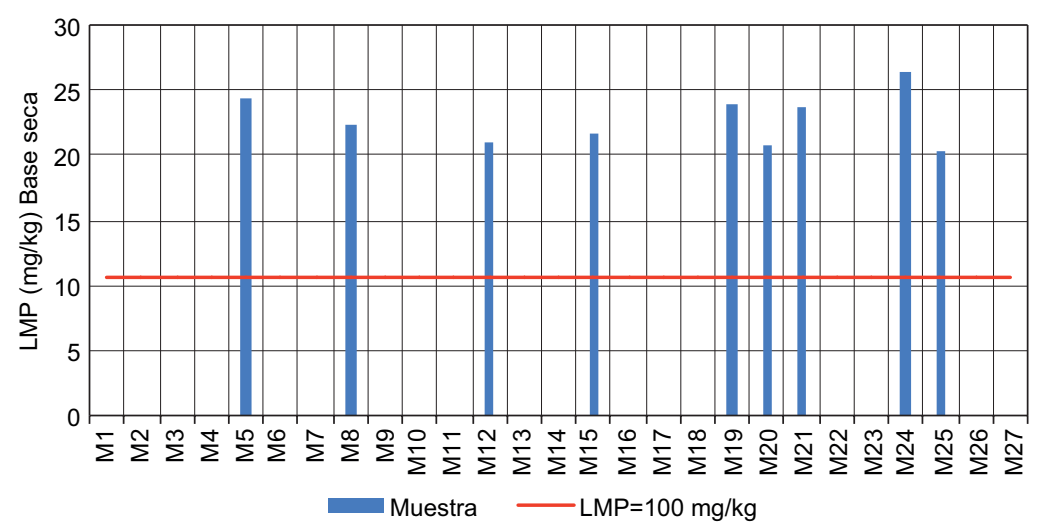

Fig. 12.Comparación de los resultados de la determinación de antimonio en muestras de jales con el límite máximo permisible (LMP)

nueve restantes $(33.3 \%)$ se encontraron cantidades de este elemento superiores al LMP (Fig. 12).

En la figura 13 se evidencia la concentración de los metales As, $\mathrm{Ba}, \mathrm{Cr}$ y $\mathrm{Sb}$ (considerados tóxicos) en cada punto de muestreo.

En una investigación realizada en el distrito minero de Guanajuato, México, Ramos-Gómez et al. (2012) demostraron que por la presencia de calcita y el bajo contenido de sulfuros fue un factor primordial para explicar la elevada estabilidad de los residuos.
El fraccionamiento de las especies metálicas fue también, en términos generales, congruente con la casi inexistente movilidad de los metales, revelando su distribución mayoritaria en fracciones con poca movilidad debido al $\mathrm{pH}$ alcalino que presentaron. Asimismo, Romero et al. (2008), en un estudio realizado en las regiones mineras de Nacozari, Sonora, Santa Bárbara, Chihuahua, Zimapán, Hidalgo y Taxco, Guerrero, concluyeron que, en la generación de drenaje ácido de los jales mineros, el factor más

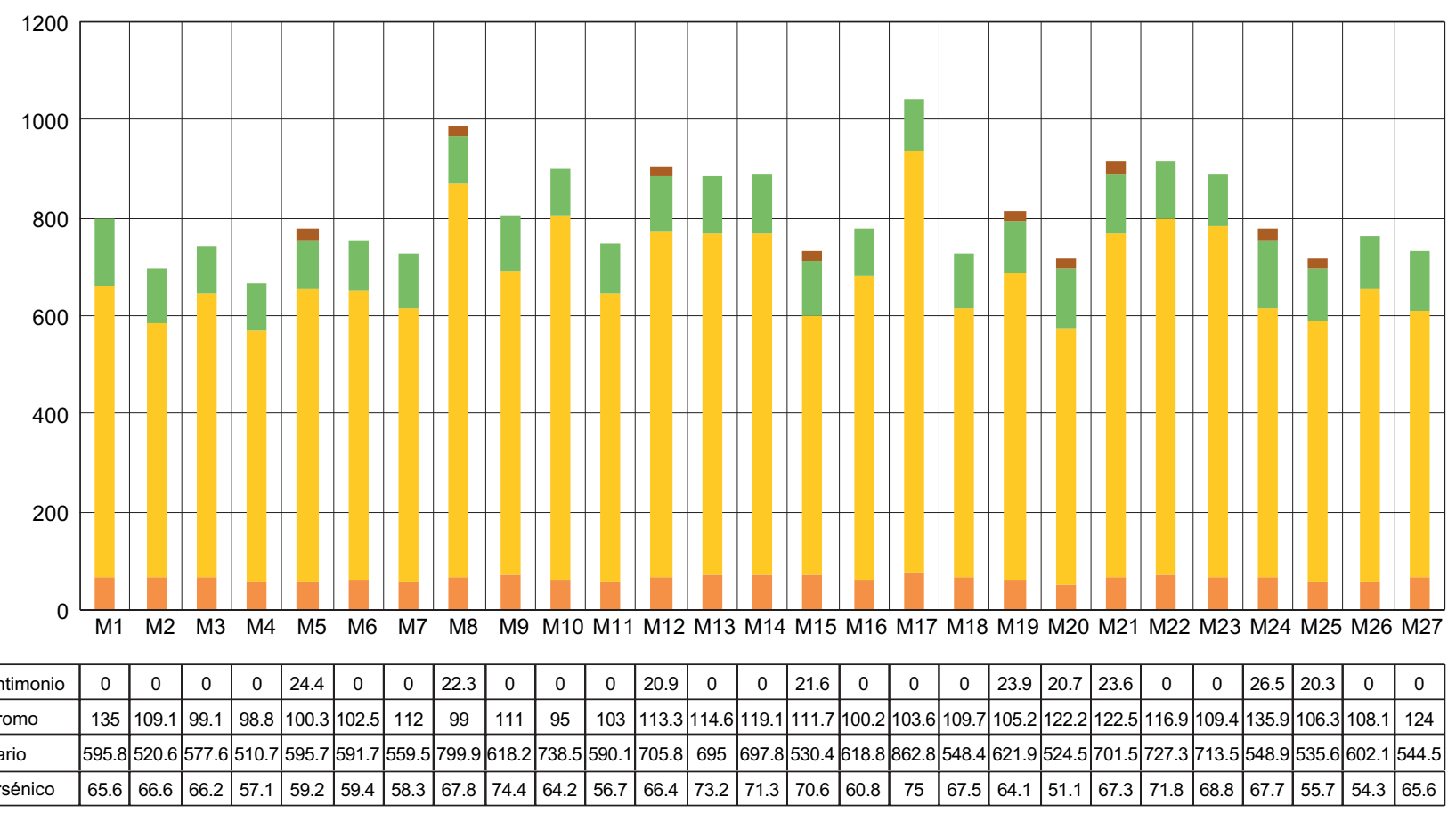

Fig. 13.Concentración de metales y metaloides en cada punto de muestreo (M) de jales 
CUADRO IV. CONCENTRACIÓN DE CROMO VI $\left(\mathrm{Cr}^{6+}\right)$ EN LAS MUESTRAS

\begin{tabular}{cccccc}
\hline Muestra & $\begin{array}{c}\text { Concentración de } \\
\mathrm{Cr}^{6+}(\mathrm{mg} / \mathrm{kg})\end{array}$ & Muestra & $\begin{array}{c}\text { Concentración de } \\
\mathrm{Cr}^{6+}(\mathrm{mg} / \mathrm{kg})\end{array}$ & Muestra & $\begin{array}{c}\text { Concentración de } \\
\mathrm{Cr}^{6+}(\mathrm{mg} / \mathrm{kg})\end{array}$ \\
\hline M-1 & 0.065 & $\mathrm{M}-10$ & 0.026 & $\mathrm{M}-19$ & 0.026 \\
M-2 & 0.018 & $\mathrm{M}-11$ & 0.01 & $\mathrm{M}-20$ & 0.037 \\
M-3 & 0.029 & $\mathrm{M}-12$ & 0.048 & $\mathrm{M}-21$ & 0.034 \\
M-4 & 0.074 & $\mathrm{M}-13$ & 0.049 & $\mathrm{M}-22$ & 0.065 \\
M-5 & 0.03 & $\mathrm{M}-14$ & 0.034 & $\mathrm{M}-23$ & 0.037 \\
M-6 & 0.017 & $\mathrm{M}-15$ & 0.006 & $\mathrm{M}-24$ & 0.034 \\
M-7 & 0.052 & $\mathrm{M}-16$ & 0.071 & $\mathrm{M}-25$ & 0.02 \\
M-8 & 0.008 & $\mathrm{M}-17$ & 0.01 & $\mathrm{M}-26$ & 0.044 \\
M-9 & 0.026 & $\mathrm{M}-18$ & 0.014 & $\mathrm{M}-27$ & 0.01 \\
\hline
\end{tabular}

importante es el geológico, especialmente la mineralogía de la mena y de la roca encajonante del yacimiento del cual provienen. La abundancia relativa de sulfuros de hierro - cuya oxidación produce acidez - y calcita - cuya disolución consume la acidez generada en los jales inalterados - determina su capacidad para la generación de drenaje ácido una vez que han ocurrido los fenómenos de oxidación/ neutralización (jales oxidados).

Por otro lado, en una evaluación realizada en Zimapán, México central, Armienta et al. (2012) encontraron que la presencia de calcita influye en la especiación, neutraliza el drenaje ácido de la mina y disminuye la movilidad de la mayoría de los metales tóxicos y metaloides. La retención de metales tóxicos y metaloides dentro de los relaves o jales probablemente se produce mediante la formación de carbonatos metálicos de baja solubilidad y la elevación del $\mathrm{pH}$, que promueve la precipitación de los hidróxidos de Fe que pueden retener $\mathrm{As}$, $\mathrm{Sb}$ y otros metales. Gavilán et al. (2017) indican que, como acción de formulación de políticas, la evaluación de riesgos debe incluirse en la regulación ambiental, utilizando para ello la estimación de la especiación química de las especies en cuestión (aquellas que son más fáciles de mover en el ambiente y que pueden ser absorbidas por el organismo).

Determinación del estado de oxidación del cromo

El análisis de determinación de metales y metaloides mostró que el cromo y el antimonio exceden los LMP establecidos en la NOM-157-SEMARNAT-2009. Severiche y Gonzáles (2013) mencionan que el cromo trivalente es esencial para los seres humanos, ya que promueve la acción de la insulina. Los derivados del cromo hexavalente (cromatos y dicromatos) por lo general son de origen antrópico $\mathrm{y}$ en altas concentraciones producen daños a los humanos y plantas. Los resultados de concentración de $\mathrm{Cr}^{6+}$ presentes en las 27 muestras se exponen en el cuadro IV.

Los valores de $\mathrm{Cr}^{6+}$ expuestos en el cuadro IV están en todos los casos por debajo de los LMP establecidos en la NOM-147-SEMARNAT/SSA1-2004 $(280 \mathrm{mg} / \mathrm{kg}$ para uso agrícola y $510 \mathrm{mg} / \mathrm{kg}$ para uso industrial). Zayed y Terry (2003) indican que el cromo se encuentra generalmente en suelos como $\mathrm{Cr}^{3+}$, en tanto que Jardine et al. (1999) mencionan que en suelos alcalinos y aireados la reducción de $\mathrm{Cr}^{6+} \mathrm{a} \mathrm{Cr}^{3+}$ es posible si el suelo contiene materia orgánica. La presa de jales Boleo Estrella presentaba una gran cantidad de vegetación (Fig. 14) así como diversos insectos. Asimismo, la superficie de la presa tenía cuarteaduras, las cuales permitían la aireación (Fig. 15).

Los resultados obtenidos en el cuadro IV concuerdan con lo reportado por los autores citados, es decir, debido a la gran cantidad de flora y fauna presente en la presa, así como a las grietas encontradas en la superficie de ésta, además del $\mathrm{pH}$ alcalino de los jales, se demuestra que existen todas las condiciones en el medio para que el cromo se encuentre principalmente como $\mathrm{Cr}^{3+}$ (a $30 \mathrm{~cm}$ ), que es la forma menos tóxica de este elemento.

\section{Estado de oxidación del antimonio}

Scheinost et al. (2006) mencionan que en suelos aireados el antimonio se encuentra principalmente como $\mathrm{Sb}^{5+}$. Si bien existen condiciones reductoras en el sitio de estudio, Mitsunobu et al. (2006) describen al $\mathrm{Sb}^{5+}$ como un estado de oxidación estable, incluso en condiciones reductoras. Asimismo, en el presente estudio se encontró presencia de $\mathrm{Sb}$, pero como $\mathrm{Sb}^{5+}$, en las muestras de jales de la presa Boleo Estrella

Okkenhaug et al. (2011) estudiaron la especiación del antimonio en el suelo cercano a la mina Xikuangshan, en China. Al analizar las muestras encontraron resultados de $\mathrm{pH}$ entre 5.81 y 7.46 , además de 


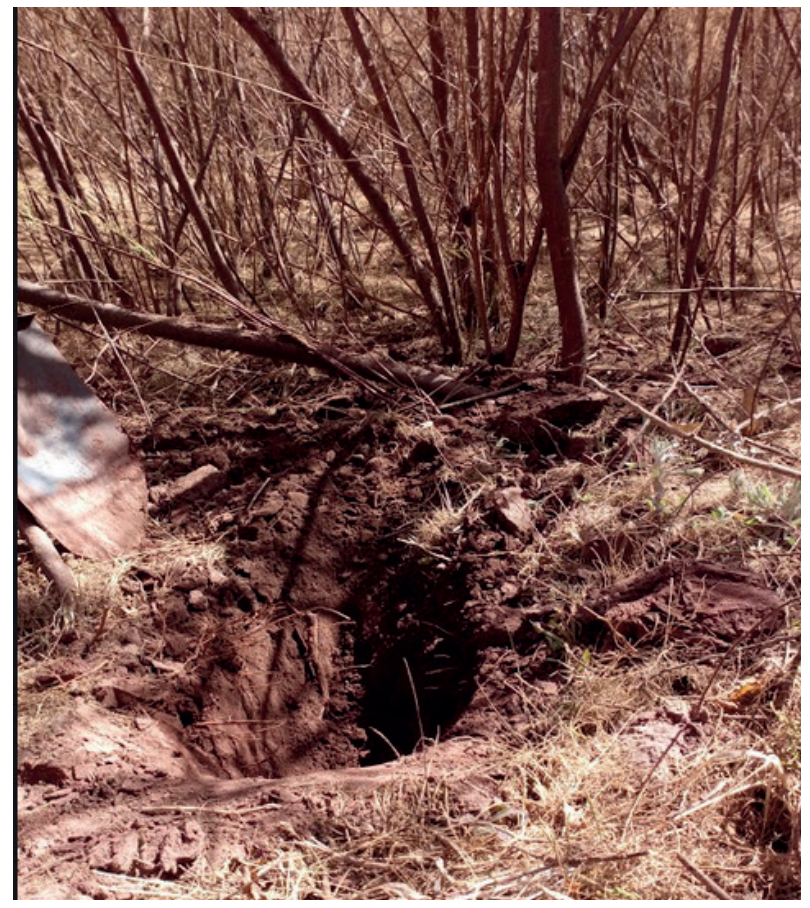

Fig. 14. Vegetación presente en la presa de Jales

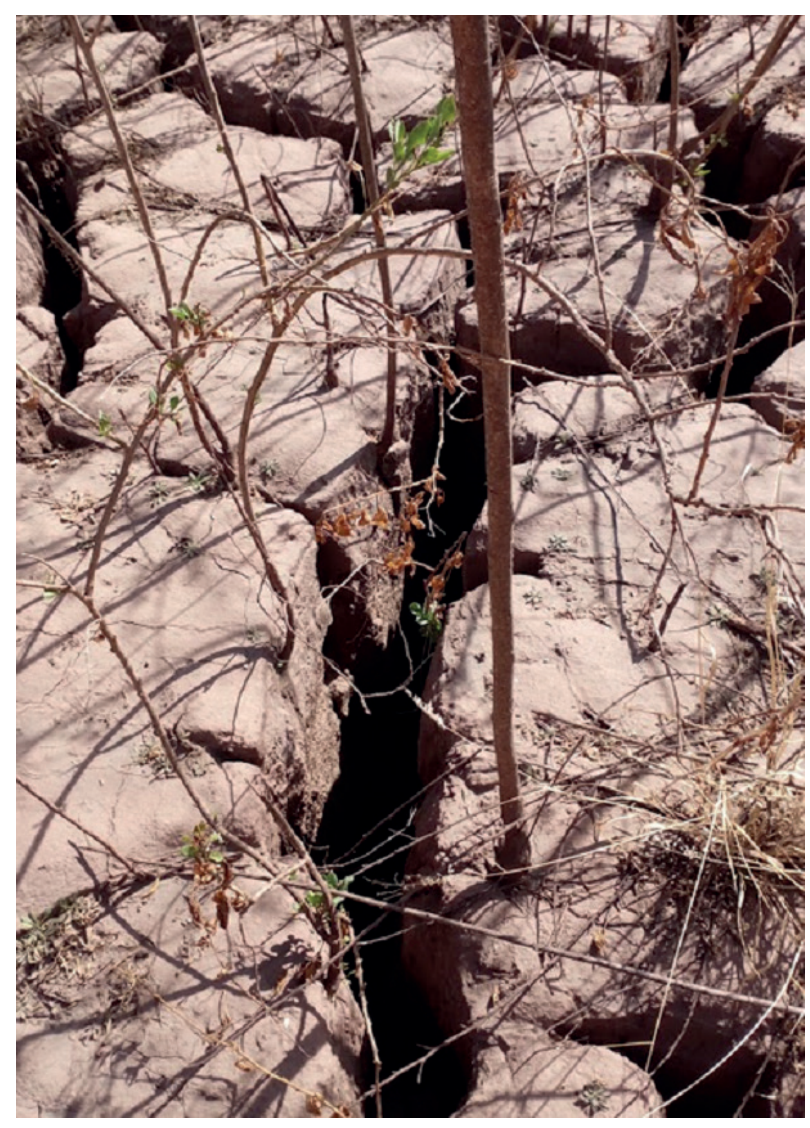

Fig. 15. Grietas presentes en la superficie de la presa de jales Boleo Estrella concentraciones de antimonio total mayores a $11.8 \mathrm{~g} / \mathrm{kg}$. Por otro lado, los resultados de especiación en el suelo de la mina, así como la vegetación presente alrededor de esta, mostraron que la especie predominante era $\mathrm{Sb}^{5+}$. Comparando las muestras de jales analizadas de la presa Boleo Estrella se obtuvieron resultados de $\mathrm{pH}$ alcalinos y en algunos casos similares a los resultados de la mina Xikuangshan, además existe la presencia de $\mathrm{Sb}$ menos toxica como $\mathrm{Sb}^{5+}$.

\section{Pruebas de movilidad}

Los resultados obtenidos en las muestras de jales de la presa Boleo Estrella demuestran que el cromo en el lixiviado, cuyo LMP es de $5 \mathrm{mg} / \mathrm{L}$, se encuentra por debajo de los $2 \mathrm{mg} / \mathrm{L}$. En el caso del antimonio, el valor registrado en el lixiviado fue de $2.1 \mathrm{mg} / \mathrm{L}$, valor que supera el LMP de $0.53 \mathrm{mg} / \mathrm{L}$ establecido en la NOM-157- SEMARNAT-2009. Esta norma también establece que cuando la concentración en el extracto de uno o varios de los elementos sea superior a los LMP, se concluye que el residuo es peligroso por la toxicidad asociada con la movilidad del elemento.

El acuífero Valle del Guadiana es la principal fuente de agua para consumo humano de la ciudad de Durango, sin embargo, La Comisión Nacional del Agua (CONAGUA 2015) ha mencionado que los principales contaminantes registrados en aguas subterráneas del Valle de Guadiana son flúor y arsénico, y que la actividad de la mina Cerro de Mercado es una de las posibles fuentes de contaminación de los acuíferos de dicho valle. Por otra parte, Alarcón et al. (2001) indican que la contaminación del agua subterránea del valle es de origen natural, causada por la composición geológica del acuífero. Asimismo, en el proceso del muestreo se observó que las membranas en las paredes de la presa Boleo Estrella son impermeables, por lo que es poco probable que el antimonio lixiviado se incorpore en los cuerpos de agua.

\section{CONCLUSIONES}

Como resultado de la determinación de posible drenaje ácido de mina y la caracterización de jales mineros provenientes de la mina Cerro de Mercado, Durango, México, y considerando la normativa mexicana, específicamente en la presa de jales Boleo Estrella, se puede concluir que en la mayoría de las muestras analizadas, las concentraciones de metales y metaloides no rebasaron los límites máximos permisibles (LMP) establecidos en la NOM-157-SEMARNAT-2009; sin embargo, las concentraciones de cromo y antimonio excedieron dichos límites. 
De acuerdo con los resultados obtenidos de la medición de $\mathrm{pH}$, la relación $\mathrm{PN} / \mathrm{PA}$ y las concentraciones de metales y metaloides, la generación de DAM es poco probable en el sitio de estudio, por lo que no se evidencia peligrosidad para las personas y el ambiente. Esto se debe a que las muestras de jales de la presa en cuestión presentan suficiente contenido de $\mathrm{CaCO}_{3}$ para neutralizar los sulfuros presentes, y en consecuencia no generan drenaje ácido. No obstante, debe prevenirse la movilización de cromo y antimonio cercanos a las áreas de la presa en las condiciones actuales. Finalmente, las pruebas de lixiviados en las muestras de jales demostraron que el cromo se encuentra por debajo de los LMP, mientras que el antimonio supera los LMP establecidos en la NOM-157- SEMARNAT-2009.

Se recomienda tomar muestras de jales de la presa Boleo Estrella de la mina Cerro de Mercado, Durango, México, a distintas profundidades, para conocer con mayor certeza el comportamiento de los metales y metaloides.

\section{AGRADECIMIENTOS}

La presente investigación fue financiada por el Programa de Becas Posdoctorales y la Dirección General de Asuntos del Personal Académico, ambos de la UNAM, y el Programa de Apoyo a Proyectos de Investigación e Innovación Tecnológica (PAPIIT, código IN113816). Un especial agradecimiento a los laboratorios de Ingeniería Sanitaria y Ambiental de la Facultad de Ingeniería, de Química Analítica del Instituto de Geofísica y Nacional de Geoquímica y Mineralogía del Instituto de Geología, de la UNAM, así como a la empresa minera Cerro de Mercado, Durango, México.

\section{REFERENCIAS}

Aduvire O. (2006). Drenaje ácido de mina. Generación y tratamiento. Instituto Geológico y Minero de España. Madrid, España, 140 pp.

Alarcón H.M.T., Flores M.I., Romero N.P., Martí D.I. y Trejo V.R. (2001). Contenido de arsénico en el Valle del Guadiana, México. Ingeniería Hidráulica en México $16,63-70$.

APHA (2003). Standard methods for the examination of water and wastewater. $4500-\mathrm{SO}_{4}-\mathrm{E}$, turbidimetric method. American Public Health Association, American Water Works Association, Water Environment Federation. Washington DC, EUA, 12 pp.
Armienta M.A., Villaseñor G., Cruz O., Ceniceros N., Aguayo A. y Morton O. (2012). Geochemical processes and mobilization of toxic metals and metalloids in an As-rich base metal waste pile in Zimapán, Central Mexico. Appl. Geochem. 27 (11), 2225-2237. https:// doi.org/10.1016/j.apgeochem.2012.01.015

Awan A.G. (2013). Relationship between environment and sustainable economic development: A theoretical approach to environmental problems. Int. J. Asian Social Sci. 3 (3), 741-761.

Bedi A., Singh B.R., Deshmukh S.K., Adholeya A. y Barrow C.J. (2018). An Aspergillus aculateus strain was capable of producing agriculturally useful nanoparticles via bioremediation of iron ore tailings. J. Environ. Manag. 215, 100-107. https://doi.org/10.1016/j. jenvman.2018.03.049

Bwapwa J.K., Jaiyeola A.T. y Chetty R. (2017). Bioremediation of acid mine drainage using algae strains: A review. South Afr. J. Chem. Eng. 24, 62-70. https:// doi.org/10.1016/j.sajce.2017.06.005

CAMIMEX (2017). Informe anual 2017. Cámara Minera de México [en línea]. https://camimex.org.mx/index. $\mathrm{php} /$ secciones $1 /$ publicaciones/informe-anual/informeanual-2017/ 8/10/2018.

CONAGUA (2015). Actualización de la disponibilidad media anual de agua en el acuífero del Valle de Guadiana (1003), estado de Durango. Comisión Nacional del Agua. Diario Oficial de la Federación, 4 de enero.

Corona-Esquivel R., Tapia Z.C., Bueno R.J.M. y Acuña P. (2003). Yacimiento de hierro Cerro de Mercado, Durango: Situación actual. Menorías AIMMGM, XXV Convención Internacional, Acapulco, Guerrero, México, 21 al 25 de octubre. CD-ROM.

Corona-Ezquivel R., Tapia-Zúñiga C., Henríquez F., Tritlla J., Morales-Insunza A., Levresse G. y Pérez-Flores E. (2009). Geología y mineralización del yacimiento de hierro Cerro de Mercado, Durango. En: Geología económica de México (Clarck K., Salas G.A. y Cubillas R., Eds.), Servicio Geológico Mexicano, México D.F., 529-535 pp.

Corona-Esquivel R., Saldaña E. y Benavides E. (2016). Mina de hierro Cerro de Mercado, Durango. Memorias. 5o Congreso Tendencias de la actividad minera en México. Durango, México, 26 de agosto. USB.

Covarrubias S.A. y Peña J.J. (2017). Contaminación ambiental por metales pesados en México: Problemática y estrategias de fitorremediación. Rev. Int. Contam. Ambie. 33, 7-21. https://doi.org/10.20937/ RICA.2017.33.esp01.01

Elvan O.D. (2013). The legal environmental risk analysis (LERA) sample of mining and the environment in Turkish legislation. Resour. Policy 38 (3), 252-257. https://doi.org/10.1016/j.resourpol.2013.03.008 
Entezari-Zarandi A., Larachi F., Beaudoin G., Plante B. y Sciortino M. (2017). Ambient mineral carbonation of different lithologies of mafic to ultramafic mining wastes/tailings a comparative study. Int. J. Greenh Gas Control 63, 392-400. https://doi.org/10.1016/j. ijggc.2017.06.016

Fraser J. (2018). Mining companies and communities: Collaborative approaches to reduce social risk and advance sustainable development. Resour. Policy (en prensa), 1-10. https://doi.org/10.1016/j.resourpol.2018.02.003

Gavilán I.C., Fernández G., Menchaca A., Barraza L.A. y Gavilán A. (2017). Policy proposal for metals speciation in tailings contaminated soils: A case study in Chihuahua, Mexico. J. Mex. Chem. Soc. 61 (1), 14-22. https://doi.org/10.29356/jmcs.v61i1.123

Gitari M.W., Akinyemi S.A., Thobakgale R., Ngoejana P.C., Ramugondo L., Matidza M., Mhlongo S.E., Dacosta F.A. y Nemapate N. (2018). Physicochemical and mineralogical characterization of Musina mine copper and New Union gold mine tailings: Implications for fabrication of beneficial geopolymeric construction materials. J. Afr. Earth Sci. 137, 218-228. https://doi. org/10.1016/j.jafrearsci.2017.10.016

Guzmán F. (2016). Impactos ambientales causados por megaproyectos de minería a cielo abierto en el estado de Zacatecas, México. Revista de Geografía Agrícola 56, 109-128. https://doi.org/10.5154/r.rga.2016.57.010

INEGI (2010). Información específica de climas. Instituto Nacional de Estadística y Geografía, México [en línea]. http://www.cuentame.org.mx/monografias/informacion/dur/territorio/clima.aspx?tema $=$ me $\& \mathrm{e}=10$. 25/10/18.

INEGI (2017). Anuario estadístico y geográfico de Durango 2017. Instituto Nacional de Estadística y Geografía, México, $501 \mathrm{pp}$.

Jardine P., Fendorf S., Mayes M., Larsen I., Brooks S. y Bailey W. (1999). Fate and transport of hexavalent chromium in undisturbed heterogeneous. Environ. Sci. Technol. 33 (17), 2939-2944. https://doi.org/10.1021/ es981211v

Johnson D.B. y Hallberg K.B. (2005). Acid mine drainage remediation options: a review. Sci. Total Environ. 338, 3-14. https://doi.org/10.1016/j.scitotenv.2004.09.002

Kastyuchik A., Karam A. y Aïder M. (2017). The effect of electro-activation and eggshell powder on the neutralization of acid mine drainage. Journal of Sustainable Mining 16 (3), 73-82. https://doi.org/10.1016/j. jsm.2017.09.002

Kefeni K.K., Msagati T.A.M. y Mamba B.B. (2017). Acid mine drainage: Prevention, treatment options, and resource recovery: A review. J. Clean. Prod. 151, 475-493. https://doi.org/10.1016/j.jclepro.2017.03.082
Kiventera J., Lancellotti I., Catauro M., Poggetto F.D., Leonelli C. y Illikainen M. (2018). Alkali activation as new option for gold mine tailings inertization. J. Clean. Prod. 187, 76-84. https://doi.org/10.1016/j. jclepro.2018.03.182

Kossoff D., Dubbin W.E., Alfredsson M., Edwards S.J., Macklin M.G. y Hudson-Edwards K.A. (2014). Mine tailings dams: Characteristics, failure, environmental impacts, and remediation. Appl. Geochem. 51, 229245. https://doi.org/10.1016/j.apgeochem.2014.09.010

Lawrence R.W. y Wang Y. (1996). Determination of neutralization potential for acid rock drainage prediction. Vancouver-Canada: Mining and mineral process engineering. University of British Columbia, Canadá, 89 pp.

Manrique-Ramírez E.J. y Rodríguez-Rosales M.G. (2015). Validación de la determinación de metales en suelo con equipo portatil de difracción de rayos X. Memorias. $2^{\circ}$ Congreso Nacional de la Asociación Mexicana de Ingeniería, Ciencia y Gestión Ambiental (AMICA). Puebla, México, 21 al 23 de octubre, CD-ROM.

Mena F.V. y Loera P.S. (1972). Guía para fabricación y control de concreto en obras pequeñas. Cámara Nacional de la Industria de la Construcción- Instituto de Ingeniería, UNAM. México D.F., México, 178 pp.

Mitsunobu S, Harada Ty Takahashi Y. (2006). Comparison of antimony behavior with that of arsenic under various soil redox conditions. Environ. Sci. Technol. 40 (23), 7270-7276. https://doi.org/10.1007/BF02839926

Navarro M.C., Pérez-Sirvent C., Martínez-Sánchez M.J., Vidal J., Tovar P.J. y Bech J. (2008). Abandoned mine sites as a source of contamination by heavy metals: A case study in a semi-arid zone. J. Geochem. Explor. 96 (2-3), 183-193. https://doi.org/10.1016/j. gexplo.2007.04.011

Nleya Y., Simate G.S. y Ndlovu S. (2016). Sustainability assessment of the recovery and utilisation of acid from acid mine drainage. J. Clean. Prod. 113, 17-27. https:// doi.org/10.1016/j.jclepro.2015.11.005

Okkenhaug G., Zhu Y-G., Lei L., Lei M., Li X. y Mulder J. (2011). Distribution, speciation and availability of antimony $(\mathrm{Sb})$ in soils and terrestrial plants from an active Sb mining area. Environ. Pollution 159 (10), 24272434 https://doi.org/10.1016/j.envpol.2011.06.028

Pape P.L., Battaglia-Brunet F., Parmentier M., Joulian C., Gassaud C., Fernandez-Rojo L., Guigner J.M., Ikogou M., Stetten L., Olivi L., Casiot C. y Morin G. (2017). Complete removal of arsenic and zinc from a heavily contaminated acid mine drainage via an indigenous SRB consortium. J. Hazard. Mater. 321, 764-772. https://doi.org/10.1016/j.jhazmat.2016.09.060

Pavoni E., Covelli S., Adami G., Baracchini E., Cattelan R., Crosera M., Higueras P., Lenaz D. y Petranich E. (2018). Mobility and fate of thallium and other 
potentially harmful elements in drainage waters from a decommissioned $\mathrm{Zn}-\mathrm{Pb}$ mine (North-Eastern Italian Alps). J. Geochem. Explor. 188, 1-10. https://doi. org/10.1016/j.gexplo.2018.01.005

Ramos-Gómez M., Avelar J., Medel-Reyes A., Yamamoto L., Godínez L., Ramírez M., Guerra R. y Rodríguez F. (2012). Movilidad de metales en jales procedentes del distrito minero de Guanajuato, México. Rev. Int. Contam. Ambie. 28 (1), 49-59.

Rashed M.N. (2010). Monitoring of contaminated toxic and heavy metals, from mine tailings through age accumulation, in soil and some wild plants at Southeast Egypt. J. Hazard. Mater. 178, 739-746. https://doi. org/10.1016/j.jhazmat.2010.01.147

Romero F.M., Armienta M.A., Gutiérrez M.E. y Villaseñor G. (2008). Factores geológicos y climáticos que determinan la peligrosidad y el impacto ambiental de jales mineros. Rev. Int. Contam. Ambie. 24 (2), 43-54.

Salinas-Rodríguez E., Hernández-Ávila J., Rivera-Landero I., Cerecedo-Sáenz E., Ma. Reyes-Valderrama I. Correa-Cruz M. y Rubio-Mihi D. (2016). Leaching of silver contained in mining tailings, using sodium thiosulfate: A kinetic study. Hydrometallurgy 160, 6-11. https://doi.org/10.1016/j.hydromet.2015.12.001

Santini T.C. y Banning N.C. (2016). Alkaline tailings as novel soil forming substrates: Reframing perspectives on mining and refining wastes. Hydrometallurgy 164, 38-47. https://doi.org/10.1016/j.hydromet.2016.04.011

Scheinost A., Rossberg A., Vantelon D., Xifra I. Kretzschmar R., Leuz A-K., Funke H. y Johnson C. (2006). Quantitative antimony speciation in shootingrange soils by EXAFS spectroscopy. Geochim. Cosmochim. Ac. 70 (13), 3299-3312 https://doi.org/10.1016/j. gca.2006.03.020

Schoenberger E. (2016). Environmentally sustainable mining: The case of tailings storage facilities. Resour. Policy 49, 119-128. https://doi.org/10.1016/j.resourpol.2016.04.009

SE (2006). Norma Mexicana NMX-AA-132-SCFI-2006. Muestreo de suelos para la identificación y la cuantificación de metales y metaloides, y manejo de la muestra. Secretaría de Economía. Diario Oficial de la Federación, México, 6 de marzo.

SE (2012). Norma Mexicana NMX-AA-013-SCFI-2006. Residuos sólidos-determinación de $\mathrm{pH}$-método de prueba. Secretaría de Economía. Diario Oficial de la Federación, México, 6 de diciembre.

SEMARNAT (2004). Norma Oficial Mexicana NOM141-SEMARNAT-2003. Que establece el procedimiento para caracterizar los jales, así como las especificaciones y criterios para la caracterización y preparación del sitio, proyecto, construcción, operación y postoperación de presas de jales. Secretaria de
Medio Ambiente y Recursos Naturales. Diario Oficial de la Federación, México, 13 de septiembre.

SEMARNAT (2007). Norma Oficial Mexicana NOM147-SEMARNAT/SSA1-2004. Que establece criterios para determinar las concentraciones de remediación de suelos contaminados por arsénico, bario, berilio, cadmio, cromo hexavalente, mercurio, níquel, plata, plomo, selenio, talio y/o vanadio. Secretaría de Medio Ambiente y Recursos Naturales. Diario Oficial de la Federación, México, 2 de marzo.

SEMARNAT (2011). Norma Oficial Mexicana NOM157-SEMARNAT-2009. Que establece los elementos y procedimientos para instrumentar planes de manejo de residuos mineros. Secretaria de Medio Ambiente y Recursos Naturales. Diario Oficial de la Federación, México, 30 de agosto.

Severiche S. y Gonzáles G. (2013). Verificación analítica para las determinaciones de cromo hexavalente en aguas por espectrofotometría. Revista Ingenierías Universidad San Buenaventura Medellín 4 (1), 22-26.

Sgier H., Macías F., Nieto J.M. y Rötting T.T.S. (2015). Diseño de una planta piloto para el tratamiento pasivo de drenaje ácido de mina. Tecnoaqua (11), 70-75.

Simate G.S. y Ndlovu S. (2014). Acid mine drainage: Challenges and opportunities. J. Environ. Chem. Eng. 2, 1785-1803. https://doi.org/10.1016/j.jece.2014.07.021

Sobek A.A., Schuller W.A., Freeman J.R. y Smith R.M. (1978). Field and laboratory methods applicable to overburdens and mine soils. EPA-600/2-78-054. U.S. Environmental Protection Agency-Industrial Environmental Research Laboratory. Cincinnati, Ohio, EUA, 33 pp.

Tost M., Hitch M., Chandurkar V., Moser P. y Feiel S. (2018). The state of environmental sustainability considerations in mining. J. Clean. Prod. 182, 969-977. https://doi.org/10.1016/j.jclepro.2018.02.051

USEPA (2007). EPA-SW-846 Test Method 6200. Field portable X-ray fluorescence spectrometry for the determination of elemental concentrations in soil and sediment. Manual. United Sates Environmental Protection. Washington DC, EUA, 32 pp.

Varrica D., Tamburo E., Milia N., Vallascas E., Cortimiglia V., De Giudici G., Dongarrà G., Sanna E., Monna F. y Losno R. (2014). Metals and metalloids in hair samples of children living near the abandoned mine sites of Sulcis-Inglesiente (Sardinia, Italy). Environ. Res. 134, 366-374. https://doi.org/10.1016/j.envres.2014.08.013 Vasilatos Ch., Koukouzas N. y Alexopoulos D. (2015). Geochemical control of acid mine drainage in abandonet mines: The case of Ermioni mine, Greece. Proced. Earth Planetary Sci. 15, 945-950. https://doi. org/10.1016/j.proeps.2015.08.151 
Volke T. y Velasco J.A. (2002). Tecnologías de remediación para suelos contaminados. Instituto Nacional de Ecología-Secretaría de Medio Ambiente y Recursos Naturales, México D.F., México, 22 pp.

Ye M., Li G., Yan P., Ren J., Zheng L., Han D., Sun S., Huang S. y Zhong Y. (2017). Removal of metals from lead-zinc mine tailings using bioleaching and followed by sulfide precipitation. Chemosphere 185, 1189-1196. https://doi.org/10.1016/j.chemosphere.2017.07.124

Zayed B. y Terry N. (2003). Chromium in the environment: factors affecting biological remediation. Plant Soil 249 (1), 139-156. https://doi. org/10.1023/A:1022504826342
Zeng B., Zhang Z. y Yang M. (2018). Risk assessment of groundwater with multi-source pollution by a longterm monitoring programme for a large mining area. Int. Biodeterior. Biodegrad. 128, 100-108. https://doi. org/10.1016/j.ibiod.2017.01.002

Zhang X., Yang H. y Cui Z. (2018). Evaluation and analysis of soil migration and distribution characteristics of heavy metals in iron tailings. J. Clean Prod. 172, 475-480. https://doi.org/10.1016/j.jclepro.2017.09.277 